\title{
The Motor Cortex Communicates with the Kidney
}

\author{
David J. Levinthal ${ }^{2,3}$ and Peter L. Strick ${ }^{1,2}$ \\ ${ }^{1}$ Pittsburgh Veterans Affairs Medical Center and Department of Neurobiology, ${ }^{2}$ Center for the Neural Basis of Cognition and Systems Neuroscience \\ Institute, and ${ }^{3}$ Division of Gastroenterology, Hepatology, and Nutrition, Department of Medicine, University of Pittsburgh School of Medicine, Pittsburgh, \\ Pennsylvania 15261
}

We used retrograde transneuronal transport of rabies virus from the rat kidney to identify the areas of the cerebral cortex that are potential sources of central commands for the neural regulation of this organ. Our results indicate that multiple motor and nonmotor areas of the cerebral cortex contain output neurons that indirectly influence kidney function. These cortical areas include the primary motor cortex (M1), the rostromedial motor area (M2), the primary somatosensory cortex, the insula and other regions surrounding the rhinal fissure, and the medial prefrontal cortex. The vast majority of the output neurons from the cerebral cortex were located in two cortical areas, M1 (68\%) and M2 (15\%). If the visceromotor functions of M1 and M2 reflect their skeletomotor functions, then the output to the kidney from each cortical area could make a unique contribution to autonomic control. The output from M1 could add precision and organ-specific regulation to descending visceromotor commands, whereas the output from M2 could add anticipatory processing which is essential for allostatic regulation. We also found that the output from M1 and M2 to the kidney originates predominantly from the trunk representations of these two cortical areas. Thus, a map of visceromotor representation appears to be embedded within the classic somatotopic map of skeletomotor representation.

\section{Introduction}

The autonomic nervous system continuously monitors and controls visceral organs to regulate the response to exercise, emotion, and environmental challenges. The sympathetic division of the autonomic nervous system is often characterized as being responsible for global "fight or flight" responses in acutely stressful situations. However, the sympathetic system also controls responses to other situations such as exposure to temperature changes, alterations in blood glucose, and exercise. Indeed, voluntary limb movement can be preceded by coordinated changes in sympathetic output which produce autonomic activity proportional to the metabolic demands of the motor task (Matsukawa et al., 1991; Vissing et al., 1991; Tsuchimochi et al., 2002). The predictive nature of some autonomic responses indicates that they cannot be solely generated by homeostatic mechanisms that are dependent on feedback from sensors in the periphery. Instead, predictive responses fit with the concept of allostatic

Received Jan. 27, 2012; revised March 15, 2012; accepted April 1, 2012.

Author contributions: P.L.S. designed research; D.J.L. performed research; D.J.L. and P.L.S. analyzed data; D.J.L. and P.L.S. wrote the paper.

This work was supported in part by funds from the Office of Research and Development, Medical Research Service, Department of Veterans Affairs, NIH Grants R01 NS24328 (P.L.S.), R01 MH56661 (P.L.S.), P40 RR018604 (P.L.S.), T32 DK063922 (D.J.L.), and a Pennsylvania Department of Health grant. The contents do not represent the views of the Department of Veterans Affairs, the U.S. government or the Pennsylvania Department of Health. We thank Dr. M. Schnell (Thomas Jefferson University, Philadelphia, PA) for the N2C strain of rabies virus, and Dr. A. Wandeler (Animal Disease Research Institute, Nepean, Ontario, Canada) for antibodies to rabies. We thank M. Page for analysis programs, and M. O'Malley, M. Watach, D. Sipula, and Dr. Isabelle Billig for expert technical assistance and advice. We also thank Dr. Richard P. Dum for help with the renal nerve surgery.

The authors declare no competing financial interests.

This article is freely available online through the J Neurosci Open Choice option.

Correspondence should be addressed to Dr. Peter L. Strick, Systems Neuroscience Institute, University of Pittsburgh School of Medicine, 4074 Biomedical Science Tower-3, 3501 Fifth Avenue, Pittsburgh, PA 15261. E-mail: strickp@pitt.edu.

DOI:10.1523/JNEUROSCI.0406-12.2012

Copyright $\odot 2012$ the authors $\quad 0270-6474 / 12 / 326726-06 \$ 15.00 / 0$ regulation (Sterling, 2012) in which a "central command" from higher brain centers generates anticipatory patterns of autonomic activity in a feedforward fashion. However, the higher brain centers that are the origin of the central command have not been fully identified.

To define potential sources of the central command, we injected rabies virus (RV) into the rat kidney and used retrograde transneuronal transport of the virus to identify the areas of the cerebral cortex that are most directly connected to this organ. We used RV as a transneuronal tracer because the virus is transported exclusively in the retrograde direction in a time-dependent fashion (Ugolini, 2010). By careful adjustment of the survival time, retrograde transneuronal transport of the virus is capable of defining multiple links in a chain of synaptically connected neurons (Kelly and Strick, 2003). We selected the kidney for this analysis for two reasons. First, neural signals to the kidney are important for rapid hemodynamic adjustments accompanying motor activity (Hohimer and Smith, 1979). Second, the kidney receives only sympathetic innervation (DiBona and Kopp, 1997). This anatomical feature restricts virus transport to one of the two neural systems dedicated to autonomic control and thus, simplifies the analysis of experimental results.

\section{Materials and Methods}

This report is based on observations from adult male Sprague Dawley rats $(250-450 \mathrm{~g})$ which received injections of RV (CVS-N2c; $1.0 \times 10^{8}, 4.5 \times$ $10^{9} \mathrm{pfu} / \mathrm{ml}$ ) into the kidney. Rabies virus was especially useful for these experiments because the animals displayed no symptoms over the prolonged survival times necessary to achieve infection of fourth- and fifthorder neurons. In general, current evidence indicates that rabies virus is transported transneuronally in all types of systems and across all types of synapses (Kelly and Strick, 2000, 2003, Hoshi et al., 2005; Ugolini, 2010). In addition, there is no evidence that the $\mathrm{N} 2 \mathrm{c}$ strain is transported more efficiently in some pathways than others. 
All procedures used in these experiments were in accordance with the Association for Assessment and Accreditation of Laboratory Animal Care and the National Institutes of Health Guide for the Care and Use of Laboratory Animals. The experimental protocol was approved by both the Institutional Animal Care and Use Committee and the Biosafety Committee. Biosafety practices conformed to Biosafety Level 2 regulations outlined in Biosafety in Microbiological and Biomedical Laboratories (Department of Health and Human Services publication No. 93-8395). Procedural details for handling virus and virus-infected animals have been published previously (Kelly and Strick, 2000).

Kidney injections. Surgeries were performed under general anesthesia (75 mg/kg Ketamine IM and Xylazine $4 \mathrm{mg} / \mathrm{kg} \mathrm{IM}$ ) and aseptic conditions. Analgesics (Buprenorphine $0.1 \mathrm{mg} / \mathrm{kg} \mathrm{SQ}$ ) were given perioperatively. The kidney (typically the left) was accessed via a paralumbar incision and then partially exteriorized. We placed four RV injections (3.5-4.0 $\mu \mathrm{l}$ each) into the kidney parenchyma using a Hamilton microsyringe with a 30 -gauge needle. The injection syringe was held in place for $30 \mathrm{~s}$. After removal of the injection needle the site was blotted with a sterile cotton swab to prevent any leakage through the injection tract. Following the injections, the wound was sutured, and the animal was returned to a cage designed for housing virus-infected animals. All animals were given free access to water and standard rat chow before and after the surgeries.

Renal nerve section. In three rats, the renal nerve was sectioned before virus injections into the kidney. Briefly, the renal nerve was identified and severed $\sim 5 \mathrm{~mm}$ from the renal hilum. We applied a $100 \%$ ethanolsoaked cotton swab to the site of the sectioned nerve to chemically destroy any remaining nerve fibers (Sahai et al., 2009). Then, following the nerve section, we injected the kidney with RV as described above.

Survival period. We varied the survival times from 78 to $134 \mathrm{~h}$. The mean survival time for third-, fourth-, and fifth-order labeling was $82 \mathrm{~h}$ $(n=3), \sim 92 \mathrm{~h}(n=5)$, and $\sim 99 \mathrm{~h}(n=7)$. At the end of the survival period, each animal was deeply anesthetized with sodium pentobarbital (100 mg/kg i.p.) and perfused transcardially with three solutions: (1) 0.1 M phosphate buffer, (2) 10\% phosphate buffered formalin, and (3) $10 \%$ phosphate buffered formalin with $10 \%$ glycerol. After perfusion, the brains and spinal cords were removed and then, stored at $4^{\circ} \mathrm{C}$ in Phospho-Tris-Azide (PTA) with 20\% glycerol.

Histological procedures. We cut serial frozen sections $(50 \mu \mathrm{m})$ of a brain block including the entire cerebral cortex and cerebellum in the coronal plane. We also cut serial frozen sections $(50 \mu \mathrm{m})$ of a spinal cord block containing the seventh thoracic segment (T7) to the second lumbar segment (L2) in the transverse plane. We stained every 10th section of the brain and every 20th of the spinal cord with cresyl violet to enable analysis of cytoarchitecture. To identify virus-infected neurons, we performed immunohistochemical reactions on free-floating sections according to the avidin-biotin peroxidase method (Vectastain, Vector Laboratories). The reactions used a mouse monoclonal antibody (M957, diluted 1:300; supplied by A. Wandeler, Animal Disease Research Institute, Nepean, Ontario, Canada) that is specific for the $\mathrm{P}$ antigen expressed by RV (Nadin-Davis et al., 2000). All reacted sections were mounted on gelatincoated glass slides, air-dried, and coverslipped with Cytoseal.

Analytic procedures. Brain and spinal cord sections were examined under the microscope for reaction product using brightfield and/or polarized light illumination. We plotted section outlines, labeled neurons, gray-white matter boundaries and other anatomic features using a computer-based charting system (MD2, Accustage). We used these plots and software written in the laboratory to create unfolded cortical maps that displayed the distribution of labeled neurons on a two dimensional surface. The procedures used to create these maps have been described in detail previously (Dum and Strick, 1991). We based the nomenclature and boundaries for cortical areas on a standard atlas of the rat brain (Paxinos and Watson, 1998).

We created a composite map of motor representation in the rat M1 and M2 based on the results from 6 studies that used intracortical stimulation to map motor responses (Hall and Lindholm, 1974; Donoghue and Wise, 1982; Neafsey and Sievert, 1982; Gioanni and Lamarche, 1985; Neafsey et al., 1986; Giszter et al., 1998). We then aligned our cortical maps of infected neurons with the composite motor map using common

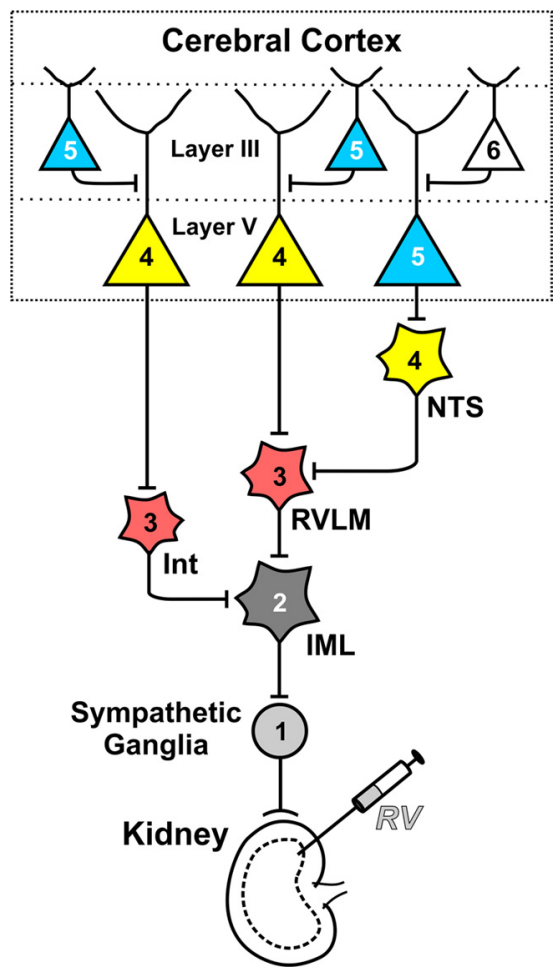

Figure 1. Retrograde transneuronal transport of RV through neural circuits that innervate the kidney. After injecting RV into the kidney, virus is transported in the retrograde direction through chains of synaptically connected neurons. This transport occurs in a time-dependent manner. Each stage of transport in the figure is numbered and color-coded: First-order, 1 (light gray); second-order, 2 (dark gray); third-order, 3 (red); fourth-order, 4 (yellow); fifth-order, 5 (blue) and sixth-order, 6 (white). IML, intermediolateral cell column: Int, spinal cord interneuron; NTS, nucleus tractus solitarius; RVLM, rostral ventrolateral medulla.

features such as the location of the bregma, and the agranular-granular border between M1 and primary somatosensory cortex.

\section{Results}

We found that the N2c strain of RV is transported centrally by sympathetic efferents following virus injections into the kidney. By careful adjustment of the survival time (78-134 h), multiple stages of replication and transneuronal transport of virus enabled the sequential infection of first-order through fifth-order neurons (Fig. 1). Animals did not display any symptoms during the extended survival periods used in these experiments. Others have reported that the CVS-11 strain of RV is not transported centrally by sympathetic efferents following virus injections into single muscles (Ugolini, 2010). The different injection sites and the use of different virus strains may explain the disparity in these results.

\section{Cortical labeling}

Infected neurons were first seen in the cerebral cortex at the same time as fourth-order neurons were found at other central sites including the nucleus of the solitary tract, periaqueductal gray, periventricular nucleus of the hypothalamus, and red nucleus. The first cortical neurons to be infected were located in Layer V, a major output layer of the cerebral cortex. By extending the survival time $8-12 \mathrm{~h}$, a subsequent stage of transneuronal transport permitted the infection of fifth-order neurons in other cortical layers (e.g., Layer III). In essence, the presence of infected neurons in Layer $\mathrm{V}$ and the absence of infected neurons in Layer III is an unequivocal marker that transport is restricted to those 
cortical neurons that are most directly connected to the kidney (Fig. 1).

In five animals, the infection of cortical neurons was limited to fourth-order neurons in Layer V. In these animals, infected neurons were predominantly $(85 \%)$ located in the hemisphere opposite to the injected kidney. Two cortical areas in the contralateral hemisphere contained the vast majority $(83 \%)$ of the infected neurons, the primary motor cortex (M1, $68 \%)$ and a region of premotor cortex, the rostromedial motor area (M2, 15\%) (Figs. $2,3)$. We found smaller numbers of infected neurons in the primary somatosensory cortex (S1,9\%), in the insula and in other regions surrounding the rhinal fissure $(7 \%)$, and in the medial prefrontal cortex (mPFC, 1\%). Thus, multiple motor and nonmotor areas of the cerebral cortex are sources of descending commands to influence the kidney. Even so, the major cortical origins of these commands are M1 and M2.

The fourth-order neurons that influence the kidney were most concentrated in distinct regions of M1 and M2. To examine the significance of this observation, we created a composite map of motor representation in M1 and M2. Then, we superimposed the location of fourth-order neurons on the motor map (Fig. 3). This analysis indicates that, within $\mathrm{M} 1$, fourth-order neurons were located largely (93\%) within regions of trunk and trunk/hindlimb representation. Within M2, fourth-order neurons were located largely within the medial portion of the forelimb representation and the lateral portion of the hindlimb representation. Neafsey et al. (1986) reported that trunk movements could be evoked following intracortical stimulation within the region of M2. Together these findings indicate that the cortical influence over the kidney originates only from a portion of the body map in M1 and M2, and specifically from the trunk representation in these two cortical areas.

Classically, regions of cingulate and insular cortex are thought to be the major origins of cortical commands to control the viscera (Iversen et al., 2000). However, these cortical areas were not heavily labeled at survival times long enough to infect fourthorder neurons (Figs. 2, 4C). It is possible that an important component of the descending control of the kidney requires "one extra synapse" and the contribution of fifth-order neurons in Layer V (Fig. 1). To explore this possibility, we extended the survival time to allow for an additional stage of transneuronal transport $(n=7)$.

In the infragranular layers (Layers V-VI) of animals with fifth-order transport (Fig. $4 B$ ), the majority of the infected neurons $(66 \%)$ were located in two cortical areas, M1 (49\%) and M2 (17\%). Smaller numbers of neurons were located in S1 (14\%), in the insula as well as in other regions surrounding the rhinal fissure $(11 \%)$, and in the mPFC (7\%). The labeled neurons in the $\mathrm{mPFC}$ were distributed among several areas including anterior cingulate, prelimbic, infralimbic, and dorsal peduncular cortex (Fig. 4D). These observations confirm that mPFC and other nonmotor areas contain output neurons that influence the sympathetic control over kidney function. Nevertheless, our findings

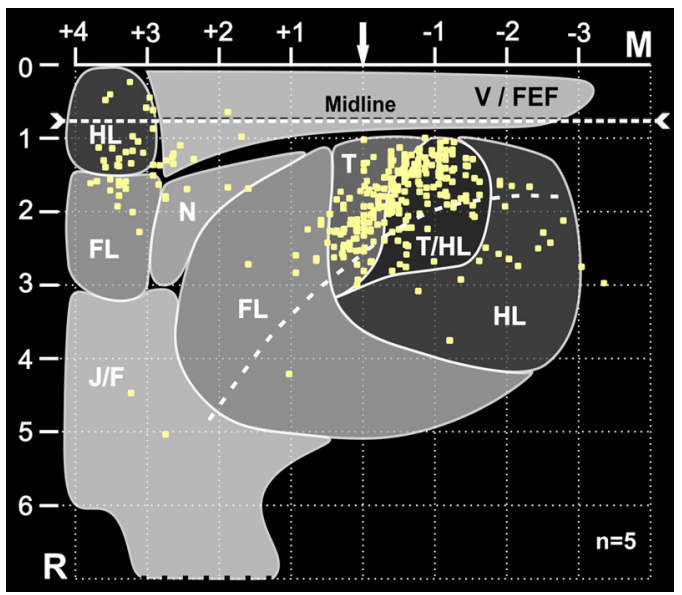

Figure 3. Fourth-order cortical neurons are located most densely in the trunk representations of M1 and M2. A composite map of fourth-order neurons in Layer V (Fig. 2B) has been superimposed on a summary map of motor representation. The motor map summarizes the results of six studies that used intracortical stimulation to evoke motor responses from regions of M1, S1, and M2 (for additional details, see Materials and Methods, Analytic procedures). The numbers on the axes represent $1 \mathrm{~mm}$ stereotaxic coordinates relative to bregma (arrow) and the intrahemispheric fissure. The "midline" is offset because it indicates the location of Layer V relative to the cortical surface. Each square represents a single labeled neuron. FL, forelimb; $H L$, hindlimb; J/F, jaw/face; M, medial; N, neck; R, rostral; T, trunk; T/HL, trunk/hindlimb overlap zone; V/FEF, vibrissae/frontal eye fields.

emphasize that the influence of these nonmotor areas is both less direct and less substantial than that of M1 and M2.

As noted above, we found labeled neurons in supragranular layers (Layers I-III) when the survival time was extended to infect fifth-order neurons. The infected neurons in the supragranular layers were located predominantly in M1 and M2 (Fig. 4A). The location of fifth-order neurons in supragranular layers was comparable to that of fourth-order neurons in Layer $\mathrm{V}$ at shorter 

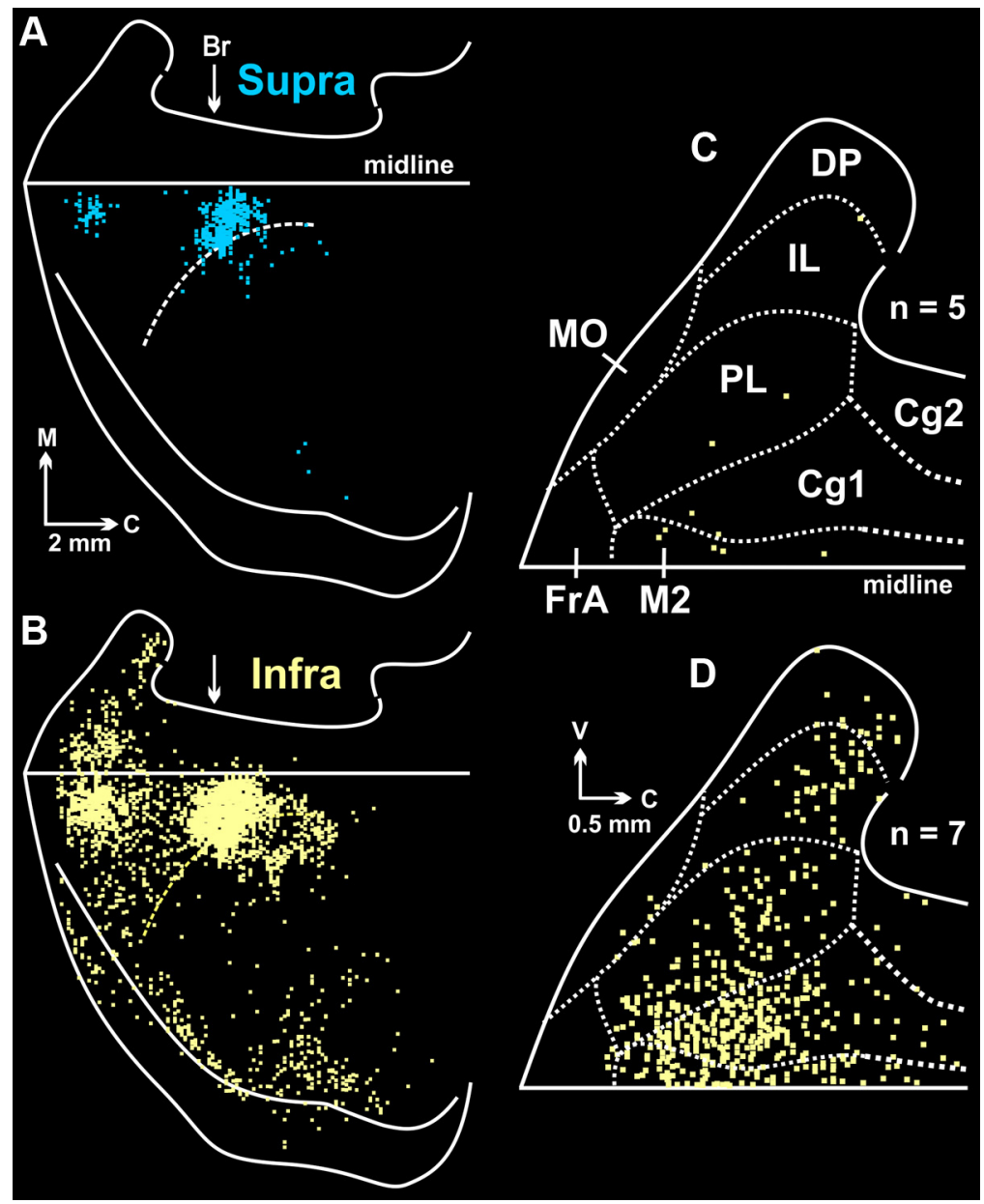

Figure 4. Cortical maps from animals with fifth-order labeling. $A$, Infected neurons in supragranular layers (Layer IV and above; Supra, blue). $\boldsymbol{B}$, Infected neurons in infragranular layers (Layers $V$ and Vl; Infra, yellow). Both maps are from the same representative animal. C, Composite map of fourth-order neurons found in cortical areas on the medial wall of the hemisphere $(n=5)$ Dashed lines indicate the cytoarchitectonic borders of specific regions. The figure is an enlarged view of the medial wall data shown in Figure 2B. $\boldsymbol{D}$. Composite map of labeled infragranular neurons in the medial wall of the hemisphere. To create this figure, we overlapped the maps of labeled infragranular neurons in animals with fifth-order labeling $(n=7$; for details see Materials and Methods, Analytic procedures). Each square represents a single labeled neuron. Br, bregma; $C$, caudal; $C \mathrm{C} 1$, cingulate cortex area 1 ; $\mathrm{Cg} 2$, cingulate cortex area 2; DP, dorsal peduncular cortex; $\mathrm{FrA}$, frontal association cortex; IL, infralimbic cortex; $\mathrm{M}$, medial; midline, midline of the hemisphere; M0, medial orbital cortex; M2, secondary motor cortex; PL, prelimbic cortex; V, ventral.

survival times (compare Fig. $4 A$ with Fig. $2 B$ ). These observations provide further support for the conclusion that M1 and M2 are the origin of the most direct and substantial cortical influence over the kidney.

\section{Control experiments}

We performed several control experiments to establish that transport of RV from kidney injections was mediated by visceromotor circuits rather than skeletomotor circuits potentially infected via spread of the virus from the injection site. In one set of animals $(n=3)$, we sectioned the left renal nerve before RV injection into the left kidney. We set the survival time of these animals long enough to enable transneuronal transport to fourth-order neurons. No infected neurons were found in the spinal cord, cerebral cortex or other regions of the CNS in these animals. These results indicate that retrograde transneuronal transport of the virus from the kidney was mediated by the renal nerve.

In another set of control experiments $(n=3)$, we injected the left kidney with RV and shortened the survival time to infect only third-order neurons. We examined the distribution of infected neurons throughout spinal segments T7 to L2 (200-250 sections/animal), as well as in supraspinal sites. Infected neurons were present in the intermediolateral cell column, at other locations in the intermediate zone of the spinal cord and in the rostral ventrolateral medulla (RVLM). On the other hand, no infected neurons were present in the ventral horn where motoneurons that innervate abdominal and back muscles are located (Brink et al., 1979). This result provides further evidence that the transneuronal transport of RV from the kidney to fourth-order neurons in Layer $\mathrm{V}$ of the cerebral cortex is mediated by visceromotor and not skeletomotor circuits. In addition, M1 and M2 are known to project to the intermediate zone of the spinal cord and to the RVLM (Miller, 1987; Ba-M'Hamed et al., 1996; Gabbott et al., 2005). Thus, the presence of infected neurons in RVLM and the intermediate zone is consistent with the proposal that third-order neurons at these sites mediate the transneuronal transport to fourthorder neurons in M1 and M2 (Fig. 1).

\section{Discussion}

Our results indicate that multiple motor and nonmotor areas of the cerebral cortex contain output neurons that indirectly influence kidney function. These cortical areas include M1, M2, S1, the insula and other regions surrounding the rhinal fissure, and mPFC. However, our findings emphasize that M1 and M2 are the origin of the most direct and substantial cortical influence over the kidney. This conclusion is consistent with prior reports on the cortical origin of inputs to the kidney (Sly et al., 1999; Cano et al., 2004).

Perhaps more importantly, we show that the output from M1 and M2 to the kidney originates predominantly from the trunk representations of these two cortical areas. This result fits with the spinal location (thoracic segments T6-T13) of renal sympathetic preganglionic neurons (Tang et al., 2004). Thus, a map of visceromotor representation appears to be embedded within the classic somatotopic map of skeletomotor representation. If this suggestion is correct, it leads to the prediction that the regions of M1 and M2 which are responsible for the control of a specific organ will be related to the spinal segments containing the neurons that innervate the organ.

Our observations are consistent with results from classic physiological experiments in monkeys which found that electrical stimulation of M1 and adjacent areas of premotor cortex evoked alterations in visceromotor function including decreases in renal blood flow (Green and Hoff, 1937; Wall and Pribram, 1950). The current results also are consistent with those from human studies in which transcranial magnetic stimulation (TMS) of human M1 and adjacent premotor areas evoked activity in skin sympathetic nerves (Silber et al., 2000). Similarly, in imaging studies, func- 
tional activation of $\mathrm{M} 1$ and the premotor cortex accompanied sympathetic activation associated with performance of a stressful task (Fechir et al., 2010). Together these observations suggest that the motor areas of the primate cerebral cortex, like those of the rat, may be important sources of central commands for the control of sympathetic function.

The involvement of M1 and M2 in autonomic control of the kidney has a number of basic science and clinical implications. For example, it is generally agreed that M1 generates and controls a variety of output parameters such as the force, speed and direction of movement (Sergio et al., 2005). M1 also is thought to be critical for the fine control of motor output. M2 is involved not only in the on-line execution of movement, but also in earlier stages of processing including the preparation to move (Wise, 1985; Hoshi and Tanji, 2007). If the visceromotor functions of M1 and M2 reflect their skeletomotor functions, then each cortical area could make a unique contribution to autonomic control of the kidney. M1 could add precision and organ-specific regulation to descending visceromotor commands, whereas M2 could add anticipatory processing which is essential for allostatic regulation.

Another consequence of locating aspects of visceromotor control in M1 and the premotor cortex is that both cortical areas receive input from two major subcortical systems - the cerebellum and basal ganglia. These subcortical systems are thought to be involved in different aspects of motor learning: the cerebellum in short-term adaptive plasticity based on error correction (Doya, 2000); the basal ganglia in the development of long-term habits based on reinforcement mechanisms (Graybiel, 2005). Thus, the involvement of M1 and the premotor cortex in visceromotor control may enable the learning mechanisms of the cerebellum and basal ganglia to shape the central command signals for autonomic function as they do the central commands for skeletomotor function. On the other hand, our results imply that dysfunction of M1, premotor cortex, cerebellum or basal ganglia could result not only in disorders of movement, but also in disorders of autonomic function. In fact, some of the autonomic difficulties faced by patients with Parkinson's disease (PD) may be due, in part, to alterations in these central circuits (Simuni and Sethi, 2008). Furthermore, deep brain stimulation in the basal ganglia, which is used to treat the motor symptoms of $\mathrm{PD}$, has been effective in treating autonomic dysfunction associated with PD (Stemper et al., 2006).

Finally, many common stress-related medical disorders, including essential hypertension and insulin resistance, have been viewed as sequelae of chronic sympathetic hyperactivity (Esler and Kaye, 1998; Flaa et al., 2008; Lambert and Lambert, 2011). Attempts to treat these disorders by conventional medical approaches have not been entirely successful. The current results suggest that M1 and the premotor cortex are potential therapeutic targets for the management of these conditions.

\section{References}

Ba-M'Hamed S, Roy JC, Bennis M, Poulain P, Sequeira H (1996) Corticospinal collaterals to medullary cadiovascular nuclei in the rat: an anterograde and retrograde double-labeling study. J Hirnforsch 37:367-375.

Brink EE, Morrell JI, Pfaff DW (1979) Localization of lumbar epaxial motoneurons in the rat. Brain Res 170:23-41.

Cano G, Card JP, Sved AF (2004) Dual viral transneuronal tracing of central autonomic circuits involved in the innervation of the two kidneys in rat. J Comp Neurol 471:462-481.

DiBona GF, Kopp UC (1997) Neural control of renal function. Physiol Rev 77:75-197.

Donoghue JP, Wise SP (1982) The motor cortex of the rat: cytoarchitecture and microstimulation mapping. J Comp Neurol 212:76-88.
Doya K (2000) Complementary roles of basal ganglia and cerebellum in learning and motor control. Curr Opin Neurobiol 10:732-739.

Dum RP, Strick PL (1991) The origin of corticospinal projections from the premotor areas in the frontal lobe. J Neurosci 11:667-689.

Esler M, Kaye D (1998) Increased sympathetic nervous system activity and its therapeutic reduction in arterial hypertension, portal hypertension and heart failure. J Autonom Nerv Sys 72:210-219.

Fechir M, Gamer M, Blasius I, Bauermann T, Breimhorst M, Schlindwein P, Schlereth T, Birklein F (2010) Functional imaging of sympathetic activation during mental stress. Neuroimage 50:847-854.

Flaa A, Aksnes TA, Kjeldsen SE, Eide I, Rostrup M (2008) Increased sympathetic reactivity may predict insulin resistance: an 18-year follow-up study. Metabolism 57:1422-1427.

Gabbott PL, Warner TA, Jays PR, Salway P, Busby SJ (2005) Prefrontal cortex in the rat: projections to subcortical autonomic, motor, and limbic centers. J Comp Neurol 492:145-177.

Gioanni Y, Lamarche M (1985) A reappraisal of rat motor cortex organization by intracortical microstimulation. Brain Res 344:49-61.

Giszter SF, Kargo WJ, Davies M, Shibayama M (1998) Fetal transplants rescue axial muscle representations in M1 cortex of neonatally transected rats that develop weight support. J Neurophys 80:3021-3030.

Graybiel AM (2005) The basal ganglia: learning new tricks and loving it. Curr Opin Neurobiol 15:638-644.

Green HD, Hoff EC (1937) Effects of faradic stimulation of the cerebral cortex on limb and renal volumes in the cat and monkey. Am J Physiol 118:641-658.

Hall RD, Lindholm EP (1974) Organization of motor and somatosensory neocortex in the albino rat. Brain Res 66:23-38.

Hohimer AR, Smith OA (1979) Decreased renal blood flow in the baboon during mild dynamic leg exercise. Am J Physiol 236:H141-H150.

Hoshi E, Tanji J (2007) Distinctions between dorsal and ventral premotor areas: anatomical connectivity and functional properties. Curr Opin Neurobiol 17:234-242.

Hoshi E, Tremblay L, Féger J, Carras PL, Strick PL (2005) The cerebellum communicates with the basal ganglia. Nat Neurosci 8:1491-1493.

Iversen S, Iversen L, Saper CB (2000) The autonomic nervous system and the hypothalamus. In: Principles of neuroscience (Schwartz JH, Jessell TM, Kandel ER, eds), pp 960-981. New York: McGraw-Hill.

Kelly RM, Strick PL (2000) Rabies as a transneuronal tracer of circuits in the central nervous system. J Neurosci Methods 103:63-71.

Kelly RM, Strick PL (2003) Cerebellar loops with motor cortex and prefrontal cortex of a nonhuman primate. J Neurosci 23:8432-8444.

Lambert EA, Lambert GW (2011) Stress and its role in sympathetic nervous system activation in hypertension and the metabolic syndrome. Curr Hypertens Rep 13:244-248.

Matsukawa K, Mitchell JH, Wall PT, Wilson LB (1991) The effect of static exercise on renal sympathetic nerve activity in conscious cats. J Physiol 434:453-467.

Miller MW (1987) The origin of corticospinal projection neurons in rat. Exp Brain Res 67:339-351.

Nadin-Davis SA, Sheen M, Abdel-Malik M, Elmgren L, Armstrong J, Wandeler AI (2000) A panel of monoclonal antibodies targeting the rabies virus phosphoprotein identifies a highly variable epitope of value for sensitive strain discrimination. J Clin Microbiol 38:1397-1403.

Neafsey EJ, Sievert C (1982) A second forelimb motor area exists in rat frontal cortex. Brain Res 232:151-156.

Neafsey EJ, Bold EL, Haas G, Hurley-Gius KM, Quirk G, Sievert CF, Terreberry RR (1986) The organization of the rat motor cortex: a microstimulation mapping study. Brain Res 396:77-96.

Paxinos G, Watson C (1998) The rat brain in stereotactic coordinates, Ed 4. San Diego: Academic.

Sahai AV, Lemelin V, Lam E, Paquin SC (2009) Central vs. bilateral endoscopic ultrasound-guided celiac plexus block or neurolysis: a comparative study of short-term effectiveness. Am J Gastroenterol 104:326-329.

Sergio LE, Hamel-Pâquet C, Kalaska JF (2005) Motor cortex neural correlates of output kinematics and kinetics during isometric-force and armreaching tasks. J Neurophysiol 94:2353-2378.

Silber DH, Sinoway LI, Leuenberger UA, Amassian VE (2000) Magnetic stimulation of the human motor cortex evokes skin sympathetic nerve activity. J Appl Physiol 88:126-134. 
Simuni T, Sethi K (2008) Nonmotor manifestations of Parkinson's disease. Ann Neurol 64 [Suppl 2]:S65-S80.

Sly DJ, Colvill L, McKinley MJ, Oldfield BJ (1999) Identification of neural projections from the forebrain to the kidney, using the virus pseudorabies. J Auton Nerv Syst 77:73-82.

Stemper B, Beric A, Welsch G, Haendl T, Sterio D, Hilz MJ (2006) Deep brain stimulation improves orthostatic regulation of patients with Parkinson disease. Neurology 67:1781-1785.

Sterling P (2012) Allostasis: A model of predictive regulation. Physiol Behav 106:5-15.

Tang X, Neckel ND, Schramm LP (2004) Spinal interneurons infected by renal injection of pseudorabies virus in the rat. Brain Res 1004:1-7.
Tsuchimochi H, Matsukawa K, Komine H, Murata J (2002) Direct measurement of cardiac sympathetic efferent nerve activity during dynamic exercise. Am J Physiol Heart Circ Physiol 283:H1896-H1906.

Ugolini G (2010) Advances in viral transneuronal tracing. J Neurosci Methods 194:2-20.

Vissing SF, Scherrer U, Victor RG (1991) Stimulation of skin sympathetic nerve discharge by central command. Circ Res 69:228-238.

Wall PD, Pribram KH (1950) Trigeminal neurotomy and blood pressure responses from stimulation of lateral cerebral cortex of macaca mulatta. J Neurophysiol 13:409-412.

Wise SP (1985) The primate premotor cortex: past, present, and preparatory. Annu Rev Neurosci 8:1-19. 